\title{
A SURVEY REPORT FOR PERFORMANCE ANALYSIS OF FINITE IMPULSE RESPONSE DIGITAL FILTER BY USING DIFFERENT WINDOW TECHNIQUES
}

\author{
Jannatul Ferdous ${ }^{1}$, Md. Sujan Ali ${ }^{2}$ \\ ${ }^{1,2}$ Assistant Professor, Department of Computer Science and Engineering, Jatiya Kabi Kazi Nazrul Islam University, \\ Trishal, Mymensingh-2220, Bangladesh
}

mjannatul@gmail.com,msujanali@gmail.com

\begin{abstract}
In the field of signal processing and communication, digital filter plays pivotal role. Digital FIR filter designed by different window techniques perform better for reducing noise from signal. In this paper, we take an overview of various window methods such as Rectangular, Triangular, Hanning, Hamming, Blackman, Kaiser and some modified windows. The magnitude responses are demonstrated for different design methods at particular cut off frequency and different filter order. All these technique have their merits and demerits. In this paper, we studied various techniques proposed earlier in literature for noise reduction from signal. This paper also provides comparative study of various filters using different window. It has been seen that Kaiser window is better for noise free signal.
\end{abstract}

Keywords: FIR filter, Rectangular window, Bartlett window, Hanning window, Hamming window, Blackman window and Kaiser window etc.

\section{INTRODUCTION}

In Linear Time Invariant (LTI) DSP systems, digital filters are significant term to modify the frequency characteristics of the input signal $x(n)$ to meet certain specific design requirements. Digital filters are broadly used for its certain advantages over Analog filters. Digital filters is better than analog filters to accomplish much improved signal to noise ratios. Finite Impulse Response (FIR) and Infinite Impulse Response (IIR) filters are the classification of digital filter. Depends on the nature of the difficulty and requirement of the desired frequency response, FIR or IIR digital filter is preferred. Flat frequency response, magnitude in pass band is frequently attractive for designing a digital filter. Linear phase is also a vital enviable characteristic of the filter. A linear phase with integer slope pilot to a simple delay in the time domain, and it decreases smallest phase distortion in the frequency domain. The FIR filter of length $M$ is expressed by the convolution of unit sample response $h(n)$ of the system and is represented by the following equation.

$$
y(n)=\sum_{k=0}^{M-1} h(k) \cdot x(n-k)
$$

Thus the coefficient of FIR filter is indicated by the impulse response of the filter. FIR filters specification include the maximum tolerable pass band ripple, maximum tolerable stop band ripple, pass band edge frequency and stop band edge frequency [1].

It is a simple and quick technique to design a FIR filter using windowing. In the window method, the desired frequency response specification $H_{d}(w)$, corresponding unit sample response $h_{d}(n)$ is determined using the following relation:

$$
\begin{gathered}
h_{d}(n)=\frac{1}{2 \pi} \int_{-\pi}^{\pi} H_{d}(w) e^{j w n} d w \\
\text { where, } H_{d}(w)=\sum_{n=-\infty}^{\infty} h_{d}(n) \cdot e^{-j w n}
\end{gathered}
$$

In general, unit sample response $h_{d}(n)$ obtained from the above relation is infinite in duration, so it must be truncated at some point say $\mathrm{n}=\mathrm{M}-1$ to yield an FIR filter of length $\mathrm{M}$ (i.e. 0 to M-1). Then $h_{d}(n)$ the impulse response of a preferred FIR filter is given by: 
$h(n)=\left\{\begin{array}{cc}h_{d}(n) & 0 \leq n \leq M-1 \\ 0 & \text { otherwise }\end{array}\right.$

The Frequency response of the desired FIR filter is

$$
H_{d}\left(e^{j w}\right)=\sum_{n=0}^{M-1} h_{d}(n) \cdot e^{-j w n}
$$

Direct truncation of $h_{d}(n)$ to $\mathrm{M}$ terms to obtain $h(n)$ leads to the Gibbs phenomenon effect which manifests itself as a fixed percentage overshoot and ripple before and after an approximated discontinuity in the frequency response due to the non-uniform convergence of the Fourier series at a discontinuity.

Thus the frequency response obtained by using above equation restrains ripples in the frequency domain. In order to lessen the ripples, $h_{d}(n)$ is multiplied by a window function $w(n)$ whose duration is finite. And it satisfies the desired magnitude response which is following.

$$
h(n)=h_{d}(n) w(n)
$$

The outline of filter specifications is depend upon the desired normalized frequencies $\left(w c, w c_{1}, w c_{2}\right)$, transition width and stopband attenuation. Both the window function and filter order are précised according to these constraints [2]. Most frequently used windows are:

1. Rectangular window:

$$
w_{R}(n)= \begin{cases}1 & 0 \leq n \leq M-1 \\ 0 & \text { otherwise }\end{cases}
$$

2. Bartlett (Triangular) window:

$w_{B}(n)= \begin{cases}1-\frac{2\left|n-\frac{M-1}{2}\right|}{M-1} & 0 \leq n \leq M-1 \\ 0 & \text { otherwise }\end{cases}$
3. Hanning window:

Casual:

$$
w_{\text {Hann }}(n)= \begin{cases}0.5-0.5 \cos \left(\frac{2 \pi n}{M-1}\right) & 0 \leq n \leq M-1 \\ 0 & \text { otherwise }\end{cases}
$$

\section{Non-Casual:}

$$
w_{\text {Hann }}(n)= \begin{cases}0.5+0.5 \cos \left(\frac{2 \pi n}{M-1}\right) \text { for }|n| 0 \leq \frac{M-1}{2} \\ 0 & \text { otherwise }\end{cases}
$$

4. Hamming window:

Casual:

$$
w_{H}(n)= \begin{cases}0.54-0.46 \cos \left(\frac{2 \pi n}{M-1}\right) & 0 \leq n \leq M-1 \\ 0 & \text { otherwise }\end{cases}
$$

Non-Casual:

$w_{H}(n)=\left\{\begin{array}{lc}0.54+0.46 \cos \left(\frac{2 \pi n}{M-1}\right) & \text { for }|n| 0 \leq \frac{M-1}{2} \\ 0 & \text { otherwise }\end{array}\right.$

5. Blackman window:

Casual:

$$
w_{\beta}(n)=\left\{\begin{array}{lc}
0.42-0.5 \cos \left(\frac{2 \pi n}{M-1}\right)+0.08 \cos \left(\frac{4 \pi n}{M-1}\right) 0 \leq n \leq M-1 \\
0 & \text { otherwise }
\end{array}\right.
$$

\section{Non-Casual:}

$w_{\beta}(n)= \begin{cases}0.42+0.5 \cos \left(\frac{2 \pi n}{M-1}\right)+0.08 \cos \left(\frac{4 \pi n}{M-1}\right) & \text { for }|n| 0 \leq \frac{M-1}{2} \\ 0 & \text { otherwise }\end{cases}$ 
6. Kaiser window:

$$
W_{K}(n)=\left\{\begin{array}{l}
\frac{I_{0}\left[\alpha \sqrt{\left(\frac{M-1}{2}\right)^{2}-\left(n-\frac{M-1}{2}\right)^{2}}\right]}{I_{0}\left[\alpha\left(\frac{M-1}{2}\right)\right]} \text { otherwise } \\
0 \leq n \leq M-1
\end{array}\right.
$$

These window methods are available for the design of filters [3][4]. In this paper we study the merits and demerits of these different approaches for performance evaluation of FIR filter.

\section{LITERATURE REVIEW}

For the performance of FIR digital filters, several of window techniques have been introduced. The DSP application demands high speed and low power digital filters. In order to meet these requirements, the order of the digital filter must be kept as small as possible. There are various sophisticated Computer Aided Design tools are available to make the digital filter fast and power efficient. Filter design and analysis tool (FDA) is one of the Computer Aided Design tool available with MATLAB which enables design of the digital filter blocks faster and more accurate. Prof.Gopal et.al. analyzed the performance of low pass FIR filter design methods for achieving minimum order [1]. In their work, they described the diverse technique for FIR filter in shortly. Among various technique, window technique showed advantageous for its simplicity. This method has been preferable by well defined equations for calculating window coefficient. For low pass filter design, Kaiser window offers very low order to meet given specification than others (Hannig, Hamming and Blackman). According to them, the best digital filter design results comes from the windowing design technique (Kaiser window) which has parameter $\alpha$ that allows adjustment between the overshoot reduction and transition region width spreading.

Era Singhal worked on design technique of low pass FIR filter using various window methods actually raised cosine windows. The stability, filter order and the filter coefficients for different window methods (Rectangular, Hamming, Hanning and Blackman) were demonstrated. To understand this technique she used window based filter equation. In this study researcher also provide the relation between frequency and magnitude for magnitude response and phase response. In her paper for simulation, she was used filter order $=20$, sampling frequency $=48000 \mathrm{~Hz}$, Cut-off frequency $=10800 \mathrm{~Hz}$. Along with divergent window methods it has been seen that filter using hamming window method is better than others. And the researcher finally proved that hamming window provide better result because its less ripples in passband, narrowest mainlobe and sidelobes, more stability and has a linear phase as compared to other window techniques [2].

Sonika Gupta, et. al. provided the information that FIR filter design by using hamming is stable as compare to rectangular and hanning windows. In their paper, they have used filter order $=61$. They have shown the magnitude and phase response of the three windows. Ripples in pass band are less in hamming as compare to other two techniques and it has high stability linear phase as compare to rectangular and hanning windows [5].

Akhilesh Chandra Bhatnagar, et. al. prepared a report is based upon the performance of different windows. The Kaiser window has been used to design a better FIR filter in terms of ripple ratio, side-lobe roll off ratio and main-lobe width. They compared the spectral characteristic of Hamming window and Kaiser Window. A modification is also introduced in Hamming window to perform better in terms of ripple ratio as compared to Kaiser using advanced Peak windowing method. They proposed an advanced peak windowing method (APW), which effectively suppresses the peak signals to the desired threshold level in case those successive peaks occur within a half of the window length. In their work, they used computer simulations. According to the simulation, they have seen that better performance can be achieved by the Hamming window over the Kaiser Window spectral characteristics. But Hamming window gives higher ripple ratio as compared to Kaiser window. They also overcome this drawback using cosh expression with hamming window. For reducing the complexity, they introduced a modified window for Hamming and Kaiser window which have a new adjusting parameter to increases the main lobe width and reduces the side lobe ratio and ripple ratio [6].

Saurabh Singh Rajput, et. al. implemented a low-pass FIR filter using Blackman window on the basis of an efficient adjustable window function. Regarding their work, the amplitude of main lobe and side lobe for a fixed bandwidth varied by changing the value of $\alpha$ of this function. They developed an adjustable window function and this window is referred to as Blackman window for $\alpha=0.16$ and Hanning window for $\alpha=0$.

$$
w(n)= \begin{cases}\frac{1-\alpha}{2}-0.5 \cos \left(\frac{2 \pi n}{M-1}\right)+\left(\frac{\alpha}{2}\right) \cos \left(\frac{4 \pi n}{M-1}\right) & 0 \leq n \leq M-1 \\ 0 & \text { otherwise }\end{cases}
$$

By selecting the value of $\alpha$ in the frequency spectrum, this window method provides higher side lobe attenuation compare to Hanning and Hamming window and the width of the mainlobe is slightly greater than Hamming window function. In some applications such as FFT, signal processing and 
measurement where higher side lobe attenuation is required compared to Hamming window this type of filters can play very significant role. Although the Blackman window function has a wider main lobe width but it used for these types of applications [7].

Suhaib Ahmed analyzed a design technique of high pass FIR filters using Hanning, Bartlett and Kaiser Windows. He demonstrated the magnitude and phase responses for different methods at particular cut off frequency $(0.4 \mathrm{rad} / \mathrm{s})$ and filter order (61). He has been shown that degree of flatness varies with the length of the filter. From the paper, the researcher concluded that for a high pass FIR filter, Kaiser Window is suitable if the transition band is of utmost importance but if he measured other parameters then Hanning window enhanced pass band attenuation. It is commonly found that Hanning window considered as a stable window as it provides good attenuation and a better transition from stop band to pass band than Bartlett and Kaiser windows [8].

Yadwinder Kumar, et. al. compared the performance of high pass FIR filters using various windowing methods (Rectangular, Hamming, Blackman and Kaiser Windows). They established only the magnitude responses of different windows at particular cut off frequency and a variety of filter order. This paper briefly describes the window and optimal techniques for design FIR filter. From the discussion, the availability of well defined equations for calculating window coefficient has made window method preferable but it has a few design flexibility. Among various windows it has been seen Kaiser window perform good magnitude response than others at different filter order [9].

Ankan Bhattacharya in his paper presented a modified window function for Finite Impulse Response (FIR) filter design with an improved frequency response. He generated a window function as like as $w(n)=a-b \cos (2 \pi(\mathrm{n}+0.1) / \mathrm{N})+c$ $\cos (4 \pi(\mathrm{n}+0.3) / \mathrm{N})-d \cos (6 \pi(\mathrm{n}+0.5) / \mathrm{N})+e \cos (8 \pi(\mathrm{n}+0.7) / \mathrm{N})$ $-f \cos (10 \pi(\mathrm{n}+0.9) / \mathrm{N})$ where, $0 \leq \mathrm{n} \leq \mathrm{N}$. And it has been used to compute the frequency response of various types of FIR filter i.e. high pass, low pass, band pass and band rejected types. In perspective of stop band attenuation and presence of ripples he also compared the frequency responses of newly generated window function with other existing windows. The compared result was justified by relevant simulations and found improved performance. According to quantized and generalized experimental results, the researcher also contrasts the frequency response of the modified window with Hamming window. For low pass filter response, modified window function comparatively much more effective attenuation than that using Hamming window. It is observe that it has resulted in much more reduced side lobes in low pass filter response. From the paper it is clear that the presence of stop band ripples is excessively high in case of Hamming window response which can be considered as a major drawback. For high pass filter, band pass and band stop filter response, presence of ripple effect much less in the modified window response than Hamming window [10].

Ankan Bhattacharya worked on another paper and presented a modified window function for FIR filter. According to quantized and generalized experimental results, the researcher also contrasts the frequency response of the modified window with Bartlett-Hann window. For low pass filter response, modified window function has much more effective attenuation and reduced side lobes than Bartlett-Hann window. But it has a major drawback which is low stop band attenuation. For high pass, band pass and band stop filter response, presence of ripple effect much less in the modified window response than Bartlett-Hann window [11].

Subhadeep Chakraborty worked on a comparative study between Blackman and hamming window. From the output of the FIR using this two window, it is clear that Blackman window technique is more powerful, perfect and useful than Hamming window. Throughout the paper it was also shown Blackman window has more terms compared to the Hamming window. More term in calculation indicates more accuracy in result. There are extra cosine terms in Blackman function. This extra term reduces the side lobes and hence increases less losing power. For FIR lowpass filter design, there are four sidelobes exists in Hamming window but for same specification and filter order, in Blackman window, there exists only two sidelobes. Similar thing found in other filters (high pass filter, band pass and band stop filter) and for this reason, the result and simulation both are more accurate in Blackman window [12].

\section{FUTURE ENHANCEMENT AND CONCLUSION}

There are various window techniques generated for analysis the performance of FIR filters. Every method has its own merits and demerits. For example, rectangular window, as the window is made longer the main lobe becomes narrower and higher, and the side lobe become more concentrated around $\mathrm{w}=0$. The main lobe width for the rectangular window is equal to $4 \pi / \mathrm{M}$. The width of the transition region depends on width of the main lobe. As the filter length $M$ increases, the main lobe becomes narrower decreasing the width of the transition region. The convolution of the desire response and side lobes give rise to the ripples in both passband and stopband. The amplitude of the ripples is dictated by the amplitude of the sidelobes. For the rectangular window, the amplitude of the sidelobes is unaffected by the length of the window. So increase the length $\mathrm{M}$ will not reduce the ripples, but increase its frequency. According to Gibbs phenomenon, maximum ripples occurs just before and after the transition band. So, the Gibbs phenomenon can be reduce by using a sudden and unexpected truncation of filter coefficients. 
If we use Bartlett window then we reduce this problem. Because the main lobe width is $8 \pi / \mathrm{M}$ or twice that of the rectangular window. The triangular window has two drawback over rectangular window, one is the transition region is high and another attenuation in stopband is less. Because of these characteristics, this window is not usually a good choice and it is not popular for FIR filter design.

Consider the Hanning window which transition region is twice in the lowpass filter. It produces small ripples in both passband and stopband. But at higher frequencies it generates greater stopband attenuation. However, at higher frequencies the Hamming window bring into being low stopband attenuation compared to of Hanning window. Because the Hamming window generates less oscillation in the sidelobes than the Hanning window, for the same main lobe width the Hamming window is generally preferred.

For the Blackmann and some modified window, the additional cosine term (compared with the Hamming and Hanning windows ) reduces the sidelobes, but incresses the main lobe width to $12 \pi / \mathrm{M}$. So, the cost increased in transition width.

On the other hand, the Kaiser window is superior to other windows, because, for given specifications its transition width is always small. By varying the parameter $\alpha$ the desire sidelobe level and main lobe peak can be achived. Further the main lobe width can be varied by varying the length $M$. That is why Kaiser window is the favorite window for designing FIR digital filter.

The future work is develop a modified window technique suitable for designing of filters with a given magnitude response and reduce the noise of signal. It has been observed that the modified window function indeed produces much more effective type of frequency responses compared to the other window. The future enhancement is to design a filter which provide accurate type of responses with reduced side lobes and comparatively less pass-band and stop-band ripples and frequency responses of the other common window types will be compared with the modified one.

\section{REFERENCES}

[1]. Prof.GopalS.Gawande,Dr.K.B.Khanchandani,T.P.Mar ode "performance analysis of FIR digital filter design techniques" International Journal of computing \& Corporate research volume 2 issue 1 January 2012.

[2]. Era Singhal "Performance Analysis of Finite Impulse Response(FIR) Filter Design Using Various Window Methods"; International Journal of Scientific Research Engineering \& Technology (IJSRET); Volume 1 Issue 5 pp 018-021 August 2012; ISSN $2278-0882$.
[3]. J.G. Proakis and D.G. Manolakis; "Digital Signal Processing: Principles, Algorithms and Applications," Pearson Prentice-Hall, fourth edition, 2011.

[4]. “Digital Signal Processing”;P.Ramesh Babu, "Scitech, third edition, 2007.

[5]. Sonika Gupta, Aman Panghal Performance "PerformanceAnalysis of FIR Filter Design by Using Rectangular, Hanning and Hamming Windows Methods" International Journal of Advanced Research in Computer Science and Software Engineering Volume 2, Issue 6, June 2012.

[6]. Akhilesh Chandra Bhatnagar, R. L. Sharma, Rajesh Kumar, "Analysis of Hammaing window using Advance Peak Windowing" International Journal of Scientific Research Engineering \& Technology (IJSRET); Volume 1 Issue 4 pp 015-020 July 2012; ISSN $2278-0882$.

[7]. Saurabh Singh Rajput, Dr S.S.Bhadauria, "Implementation of FIR filters using efficient window function and its application in filtering a speech signal" International Journal of Electrical, Electronics and Mechanical Controls (IJEEMC),vol.1,1November 2012.

[8]. Suhaib Ahmed, "Design Analysis of High Pass FIR Filters Using Hanning, Bartlett and Kaiser Windows" International Journal of Advanced Research in Computer Science and Software Engineering; Volume 2, Issue 11, November 2012.

[9]. Yadwinder Kumar, Er.Gurpreet Singh Walia "Review: Performance Analysis of FIR Digital High Pass Filters" International Journal of Engineering Research and Applications (IJERA) Vol. 3, Issue 2, pp.1012-1015, March -April 2013; ISSN: 2248-9622

[10]. Ankan Bhattacharya; "A modified window function for FIR filter design with an improved frequency response and its comparison with the Hamming window"; ISSN: 2278 - 7798; International Journal of Science, Engineering and Technology Research (IJSETR); Volume 2,Issue 5, May 2013.

[11]. Ankan Bhattacharya; "A modified window function for FIR filter design with an improvedfrequency response and its comparison with the Bartlett-Hann window"; ISSN: 2278 - 7798; International Journal of Science, Engineering and Technology Research (IJSETR); 
Volume 2,Issue6, June 2013.

[12]. Subhadeep Chakraborty. "Advantages of Blackman Window over Hamming Window Method for designing FIR Filter" International Journal of Computer Science \& Engineering Technology (IJCSET); Vol. 4 No. 08 Aug 2013; ISSN : 2229-3345

\section{BIOGRAPHIES}

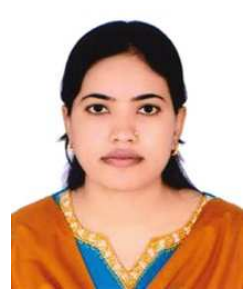

Jannatul Ferdous: She achieved her B.Sc.(Hons.) and M.Sc. degree in Applied Physics, Electronics \& Communication Engineering from Islamic University, Kushtia, Bangladesh. At present, she is an $\mathrm{PhD}$ researcher in the Department of Computer Science \& Engineering, University of Rajshahi, Rajshahi, Bangladesh. She has nearly eight years teaching experience in electronics and communication related subjects. She has published two research paper in International Conference Proceedings and eight journal articles in leading national and International Journals. Her research area in the field of Signal Processing.

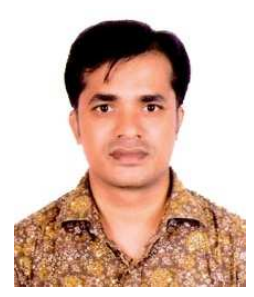

Md. Sujan Ali: $\mathrm{He}$ received his B.Sc.(Hons.) and M.Sc. degree in Applied Physics, Electronics \& Communication Engineering from Islamic University, Kushtia, Bangladesh. He is working as an Assistant Professor in the Department of Computer Science and Engineering, Jatiya Kabi Kazi Nazrul Islam University, Mymensingh, Bangladesh. Currently he is persuing $\mathrm{PhD}$ degree on Brain Signal Processing under the faculty of Engineering, Department of Computer Science \& Engineering, University of Rajshahi, Rajshahi, Bangladesh. 